\title{
Role of Aquaporin-3 in Intestinal Injury Induced by Sepsis
}

\author{
Yeke Zhu, ${ }^{a, b, \#}$ Yefeng Wang, ${ }^{a, b, \#}$ Wengbin Teng, ${ }^{a, b}$ Yue Shan, ${ }^{a}$ Shenghua Yi, ${ }^{a}$ Shengmei Zhu, ${ }^{*, c}$ and \\ Yuhong $\mathrm{Li}^{*}, a, b$ \\ ${ }^{a}$ Department of Anesthesiology, Shaoxing People's Hospital (Shaoxing Hospital of Zhejiang University); No. 568 \\ Zhongxing North Road, Shaoxing 312000, Zhejiang, China: ${ }^{b}$ Clnical Research Center, Shaoxing People's Hospital \\ (Shaoxing Hospital of Zhejiang University); No. 568 Zhongxing North Road, Shaoxing 312000, Zhejiang, China: and \\ ${ }^{c}$ Department of Anesthesiology, the First Affiliated Hospital of School of Medicine, Zhejiang University; No. 79 \\ Qingchun Road, Hangzhou 310000, Zhejiang, China. \\ Received January 28, 2019; accepted June 6, 2019
}

Aquaporin-3 (AQP3) is expressed in various parts of the intestine, where it regulates the proliferation and migration of intestinal epithelial cells and the transport of glycerol and hydrogen peroxide. Our study aimed to investigate the effect on the expression of AQP3 of intestinal injury in septic mice and whether oral administration of glycerol can reduce intestinal epithelial injury and barrier disorder by acting as a partial substitute for the function of AQP3. We established a sepsis model by cecal ligation and perforation (CLP) in mice. Sepsis induced intestinal injury, as demonstrated by the disordered destruction of the morphology of the intestinal mucosa, time-dependent increases in Chiu's score $(p<0.05)$, significantly increased $(p<0.05)$ plasma concentrations of determination of the levels of diamine oxidase (DAO) and intestinal fatty acidbinding protein 2 (FABP2), and time-dependent downregulation of the expression of AQP3 and occluding $(p<0.05)$. While the administration of oral glycerol partially ameliorated the sepsis-induced injury of the intestinal mucosa, as shown by the partial recovery of the morphological structure, with decreased Chiu's score, decreased plasma concentrations of DAO and intestinal-type FABP2, upregulated expression of occludin and decreased mortality rate (Sepsis vs. Sepsis + Glycerol, $p<0.05$ ). The results showed that the expression levels of AQP3 and occludin were downregulated after septic intestinal injury, while treatment with glycerol, which acts as a substitute for AQP3, partly ameliorated intestinal injury and improved the survival rate. This preliminary experiment suggests that $A Q P 3$ may protect the intestinal tract against the effects of sepsis.

Key words sepsis; aquaporin-3; glycerol; intestinal injury

\section{INTRODUCTION}

Sepsis is a life-threatening syndrome with multiple organ dysfunction syndrome (MODS) due to the host's dysfunctional response to infection, ${ }^{1)}$ and it is considered a public health issue requiring substantial attention. Severe sepsis affects more than a million people, including approximately $20 \%$ of all intensive care unit (ICU) patients, and the total cost to the health care system is approximately \$24 billion. ${ }^{2)}$ Epidemiological data have revealed that the incidence of sepsis has increased from 3.8 to 8 per 1000 as the population has aged, while the sepsis-related mortality rate decreased from 10 to $8.3 \%{ }^{3)}$ Recently, an aging population has led to an increase in the number of patients admitted to ICUs. However, this increase in ICU admissions has led to an increase in the prevalence of sepsis. ${ }^{4)}$ Although rapid diagnosis and reasonable nursing measures have partly reduced the mortality rate of sepsis, there is still a lack of effective treatments for sepsis. In addition, the complexity of the disease and the long-term treatment leads to undesirable consequences, such as physical, mental and functional disorders. ${ }^{5)}$ Therefore, it is important to further investigate the treatment of sepsis to promote early recovery.

The intestinal tract is the largest repository of bacteria in the body and is considered the initiator of sepsis or critical

\footnotetext{
\# These authors contributed equally to this work.

illness. ${ }^{6,7)}$ A barrier formed by intestinal epithelial cells prevents bacteria and endotoxins from moving from the gut into the bloodstream and acts as the body's largest immune organ, responding to the body's inflammatory status. ${ }^{8)}$ The mechanisms of action of the intestinal mucosal barrier are very complicated, and they may be related to an intestinal mechanical barrier, local biological factors, chemical factors and immune function; however, further research is needed.

Aquaporins (AQPs) was one kind of the membrane channel proteins which could transport water molecules selectively and efficiently. Studies showed that AQPs expressed abundantly in intestinal epithelial cells, and AQPs was involved in the secretion of intestinal mucus. Aquaporin-3 (AQP3) is hydrophobic, weighs $30 \mathrm{kDa}$ and belongs to the aquaporin family, which is expressed in various parts of the intestine, ${ }^{9}$ regulating the proliferation and migration of intestinal epithelial cells and transporting glycerol and hydrogen peroxide. In recent years, an increasing number of studies have found that the abnormal expression of AQPs during sepsis induces injuries to multiple important organs, including the heart, brain, kidney, and lung, indicating that AQPs may be an important biomarker of sepsis. ${ }^{10)}$ The intestine is the primary organ involved in sepsis, and intestinal epithelial barrier disorders have long received concentrated attention. Because AQP3 is abundantly expressed in the small intestine, whether it protects the intestinal mucosa barrier, especially the damaged intestinal mucosa during sepsis, remains unclear. Based on previous studies, we hy- 
pothesized that AQP3 has a protective effect against intestinal mucosal damage during sepsis.

In this study, to verify this hypothesis, a sepsis mouse model was established by cecal ligation and perforation. The mucosal structure, barrier damage and expression of AQP3 in the intestinal were detected. In addition, the effects of oral glycerol on intestinal injury and survival were observed. The results preliminarily verified that the expression of AQP3 in the intestinal tract plays an important role in protecting the intestine during sepsis.

\section{MATERIALS AND METHODS}

Animals and Groups The study protocol and the experimental procedure were approved by the Animal Research Ethics Committee of Zhejiang University (P.R. of China, approval number ZJU 12956), and the study was conducted in adherence with the Guide for Care and Use of Laboratory Animals. One hundred twenty-eight Balb/c male mice (6-8 weeks, 20-25g) were purchased from the Animal Research Center (Zhejiang Medical Academy, Zhejiang Province, China, license number: ZJU2015-207-10) and acclimatized for two weeks before undergoing experimentation. The mice were maintained under normal conditions in the experimental animal center of Shaoxing People's Hospital (license number SYXK (Zhe) $2017-0007$ ) at $20 \pm 1^{\circ} \mathrm{C}$ and 50 to $60 \%$ humidity, with a $12: 12$-hour light/dark cycle, a ventilation rate of 8 times in $15 \mathrm{~h}$, and free access to food.

Sepsis Model and Grouping We established a sepsis model by cecal ligation and perforation (CLP) in mice. ${ }^{11)}$ Using a randomized block design, the mice were subdivided into the following 4 groups: sham operation (Sham, $n=6$ ), sham operation with oral glycerol (Sham + glycerol, $n=6$ ), sepsis (Sepsis, $n=30$, among this group, 24 for the time dependent sepsis group) and sepsis with oral glycerol (Sepsis + glycerol, $n=6)$. All mice were anesthetized via intraperitoneal injection of $10 \%$ chloral hydrate $(400 \mathrm{mg} / \mathrm{kg})$ before undergoing surgical procedures, and their body temperatures were maintained at $36-38^{\circ} \mathrm{C}$ by a heating pad. After skin preparation and disinfection, all mice were subcutaneously injected with $0.5 \%$ ropivacaine $(1 \mathrm{~mL} / \mathrm{kg})$ and then a $1.5 \mathrm{~cm}$ incision was made through the midline. Subsequently, $50 \%$ of the cecum was ligated and then perforated by a single puncture with a 21-gauge needle in the least vascularized area. The cecum was then gently squeezed to extrude a small amount of feces from the perforation site. We attempted to standardize the quantity of feces extruded among the mice. The quantity of extruded feces was limited (a small droplet, approximately $1 \mathrm{~mm}$ in diameter) and consistent among all mice. Then, the cecum was replaced in the abdominal cavity, and the incision was closed in two layers via continuous suturing with 4.0 silk. The Sham group was treated in an identical manner, but without cecal ligation and puncture. Each mouse received a subcutaneous injection of $1 \mathrm{~mL}$ of normal saline to replenish fluids after the surgery. All animals were tolerated the procedures well, and no additional sedatives were necessary to maintain their immobility; no antibiotics were administered. The mice were given free water or glycerin before surgery. Six hours after the operation, the mice were given $0.2 \mathrm{~mL}$ of water or glycerin every $6 \mathrm{~h}$.

Glycerol Replacement Experiments The mice in the oral glycerol groups received supplemental oral glycerol $(2 \%$ in drinking water, Hunan Erkang Pharmaceutical, China). ${ }^{12}$ ) After treatment with oral glycerol, their survival and intestinal histology were assessed.

Blood Plasma and Intestinal Tissues Collection At 0 , 6, 12, and $24 \mathrm{~h}$ after CLP, the mice were killed, and blood samples were taken via eyeball extraction. The blood samples were centrifuged at $3000 \times \boldsymbol{g}$ and $4^{\circ} \mathrm{C}$ for $15 \mathrm{~min}$, and the plasma was separated from the blood and stored at $-80^{\circ} \mathrm{C}$ for use in the subsequent assays. After the mice were sacrificed by cervical dislocation, a 0.5 to $1.0 \mathrm{~cm}$ long segment of the distal ileum ( $3 \mathrm{~cm}$ proximal to the ileocecal valve) was excised and tested in duplicate. One part of the segment was fixed immediately using 4\% formaldehyde for further histological and immunohistochemistry analyses. The intestinal mucosa was harvested from the other part of the segment and immediately frozen in liquid nitrogen for $2 \mathrm{~h}$ before being transferred for storage at $-80^{\circ} \mathrm{C}$ prior to further analysis.

Mortality Rate The survival studies were conducted with 20 animals; there were 4 animals in each of the following groups: Sham, Sham + glycerol, Sepsis, and Sepsis + glycerol. Mice were observed every $12 \mathrm{~h}$ after the operation, and any deaths were recorded. The mortality and $84 \mathrm{~h}$ survival rates after surgery were investigated through close follow-up; the estimated probability was analyzed by Kaplan-Meier survival curves.

Morphological Examination of the Intestine The intestinal tissues were embedded in paraffin wax, sectioned into 5- $\square \mathrm{m}$ thick slices, stained with hematoxylin and eosin (H\&E), processed according to standard methods, examined under a microscope (Nikon, Tokyo, Japan) and analyzed by two investigators who were blinded to the experimental protocols to prevent bias. Histological mucosal damage was classified by graded criteria based on Chiu's scoring method ${ }^{13)}$ according to changes in the villus and glands of the intestinal mucosa, as follows: grade 0, normal mucosal villi; grade 1, development of subepithelial Gruenhagen's space at the apex of the villus, often with capillary congestion; grade 2, extension of the subepithelial space with moderate lifting of the epithelial layer from the lamina propria; grade 3, massive epithelial lifting down the sides of villi, possibly with a few denuded tips; grade 4, denuded villi with lamina propria and dilated capillaries exposed, possibly with increased cellularity of the lamina propria; and grade 5, digestion and disintegration of the lamina propria, hemorrhage, and ulceration. A minimum of 5 randomly chosen fields from each sample were evaluated and averaged to determine the mucosal damage.

Determination of the Levels of Diamine Oxidase (DAO) and Intestinal-Type Fatty Acid-Binding Protein (FABP2) in the Plasma by Enzyme-Linked Immunosorbent Assay (ELISA) We use spectrophotometry (SpectraMax Plus, Molecular Devices, Sunnyvale, CA, U.S.A.) to quantify the levels of DAO and intestinal-type FABP2 (both from CloudClone Corp., U.S.A.) in the plasma with ELISAs, which were performed according to the manufacturer's instructions. The experiment was repeated three times, and the results are presented as the mean values.

Immunohistochemical (IHC) Analysis of AQP3 The slides used in the IHC analyses were deparaffinized in xylene, rehydrated with graded concentrations of ethanol, immersed in $3 \%$ hydrogen peroxide solution for $10 \mathrm{~min}$, treated with 
Table 1 The Sequences of the Primers Used in the Semiquantitative qRT-PCR

\begin{tabular}{|c|c|c|}
\hline Gene & Forward primers & Reverse primers \\
\hline GAPDH & 5'-ACATCATCCCTGCATCCACT-3' & 5'-CCTGCTTCACCACCTTCTTG-3' \\
\hline TNF- $\alpha$ & 5'-AGCCAGAAAGCATGATCCG-3' & 5'-CTGATGAGAGGGAGGCCATT-3' \\
\hline IL-6 & 5'-CCTCTGGTCTTCTGGAGTACC-3' & 5'-GTCCTTAGCCACTCCTTCTGT-3' \\
\hline $\mathrm{INF}-\gamma$ & 5'-CGCTACACACTGCATCTTGG-3' & 5'-TCCTTTTGCCAGTTCCTCCA-3' \\
\hline AQP3 & 5'-GAATCGTTGTGGGGAGATGC-3' & 5'-CAAGATGCCAAGGGTGACAG-3' \\
\hline Occludin & 5'-TATGGCGGAGAGATGCATGT-3' & 5'-CCAAAGAGCCCTGTCCCATA-3' \\
\hline
\end{tabular}

citrate buffer $(\mathrm{pH} 6.0)$ at $95^{\circ} \mathrm{C}$ for $25 \mathrm{~min}$ for heat-induced antigen retrieval and cooled at room temperature for $60 \mathrm{~min}$. After each incubation step, the slides were washed with phosphate buffered saline (PBS) ( $\mathrm{pH}$ 7.4). Then, the slides were incubated separately with anti-AQP3 antibody (dilution $1: 1200$, Abcam, England) overnight at $4{ }^{\circ} \mathrm{C}$ in a humid atmosphere. Immunostaining was performed using 3,3'-diaminobenzidine (DAB) (Beyotime Institute of Biotechnology, Shanghai, China). The slides were subsequently counterstained with Mayer's hematoxylin solution (Beyotime Institute of Biotechnology), dehydrated with graded concentrations of ethanol, cleared with xylene, and mounted for visualization using bright-field microscopy.

Western Blotting Analysis of the Protein Expression Levels of AQP3 and Occludin Intestinal tissue was milled in liquid nitrogen and lysed in ice-cold radioimmunoprecipitation assay buffer (Beyotime Institute of Biotechnology) containing $0.01 \%$ protease and phosphatase inhibitor (Sigma, Shanghai, China) before being incubated on ice for $30 \mathrm{~min}$. Then, the cells were centrifuged at $12000 \times \boldsymbol{g}$ and $4^{\circ} \mathrm{C}$ for $30 \mathrm{~min}$. The protein concentration in the supernatant fluid was measured by a BCA protein assay. Equal amounts of protein were subjected to sodium dodecyl sulfate-polyacrylamide gel electrophoresis (SDS-PAGE) and transferred to polyvinylidene fluoride (PVDF) membranes. The transferred proteins were blocked with 5\% skim milk for an hour. After blocking, the membranes were probed with anti-AQP3 (1:1000), anti-occludin $(1: 1000)$ and anti- $\beta$-actin $(1: 5000)$ primary antibodies (Cell Signaling Technology Inc., NY, U.S.A.) overnight at $4^{\circ} \mathrm{C}$ and then blotted with the respective secondary antibodies (1:5000, Sigma Chemical Co., St. Louis, MO, U.S.A.). The membranes were analyzed using super ECL detection reagent (Beyotime Institute of Biotechnology).

Quantitative (q)RT-PCR Analysis of AQP3, Occludin and Inflammatory Factors Total RNA was extracted from the intestinal tissue using TRIzol reagent (Invitrogen, Tokyo, Japan) after the tissue was milled in liquid nitrogen. Total RNA ( $\leq 1000 \mathrm{ng}$ ) was reverse-transcribed into cDNA in a $20-\mu \mathrm{L}$ reaction volume using a Double-Strand cDNA Synthesis Kit (TaKaRa, Dalian, China). PCR was performed with $1 \mu \mathrm{L}$ of cDNA as a template in triplicate using the Power SYBR Green PCR Master Mix (TaKaRa) and the ABI Step One Plus System (Applied Biosystems, Warrington, U.K.). The total volume $(20 \mu \mathrm{L})$ of each reaction consisted of $10 \mu \mathrm{L}$ of SYBR Green QPCR Master Mix, $3 \mu \mathrm{L}$ of $\mathrm{ddH}_{2} \mathrm{O}, 1 \mu \mathrm{L}$ of cDNA, and $10 \mu \mathrm{M}$ each forward and reverse primer. The cycling conditions were as follows: $95^{\circ} \mathrm{C}$ for $10 \mathrm{~min}$ (activation), followed by 40 cycles at $95^{\circ} \mathrm{C}$ for $10 \mathrm{~s}, 60^{\circ} \mathrm{C}$ for $20 \mathrm{~s}$, and $72^{\circ} \mathrm{C}$ for $90 \mathrm{~s}$. Glyceraldehyde-3-phosphate dehydrogenase (GAPDH) was utilized as the housekeeping gene, and the independent experiments were repeated at least three times. The specific primers (based on the mouse sequences) used are shown in Table 1.

Statistical Analysis All experimental data are expressed as the means \pm standard deviation (S.D.); all data were plotted and analyzed using Graph Prism 7 software. Multiple comparisons of survival among groups were performed using the Kaplan-Meier method. Differences between groups were analyzed with one-way ANOVA. For data with homogeneity of variance, pairwise comparisons were performed using the LSD, and for data with inhomogeneity of variance, the Dunnett T3 test was used. Statistical significance was defined as $p<0.05, p<0.01$, and $p<0.001$.

\section{RESULTS}

Glycerol Replacement Experiments Partly Reduce the Mortality Rate After CLP operation, mice gradually showed decreased activity, tiredness, hair shaft phenomenon, abdominal exudate, diarrhea or mucus excretion. Eighty four hours mortality rates in the Sham, Sham with oral glycerol, Sepsis, and Sepsis with oral glycerol groups were 0, 0, 100, $80 \%$, respectively. Most of the deaths occurred within the first $48 \mathrm{~h}$. The mortality rate was significantly higher in the Sepsis with oral glycerol group than in the Sham or Sham with oral glycerol groups $(p<0.001)$; however, the mortality rate in the Sepsis with oral glycerol group was lower than that in the Sepsis group $(p<0.05)$ as shown in Fig. 1.

The Expression Levels of Tumor Necrosis Factor (TNF)- $\alpha$, Interleukin (IL)-1 $\beta$ and IL-6 in Intestinal Tissue Increased after CLP To verify the sepsis model and intestinal mucosa injury, qPCR was conducted to evaluate the changes in expression levels of the cytokines TNF- $\alpha$, IL- $1 \beta$ and IL-6. As shown in Fig. 2, the gene expression levels of IL-1 $\beta$ and IL- 6 increased progressively with increased time after CLP and peaked $24 \mathrm{~h}$ after CLP $(p<0.05)$. In contrast, the gene expression of TNF- $\alpha$ showed a different but still increasing trend and peaked $6 \mathrm{~h}$ after CLP.

Sepsis-Induced Intestinal Barrier Injury To evaluate the functioning of the intestinal barrier during sepsis, we examined H\&E-stained sections of the intestinal mucosa to observe the morphological changes in the intestine. The histopathological analysis showed integrated intestinal mucosa and the compactly arrayed epithelium in mice killed immediately after CLP $(0 \mathrm{~h})$. In contrast, with increasing time after CLP, the mucosal function damage became more serious, with shrinkage of the intestinal villi and expansion of the intestinal space; the microvilli became thin and short, and some microvilli were ruptured (Fig. 3A). As the time post-CLP increased, Chiu's score progressively increased, with a significant time- 


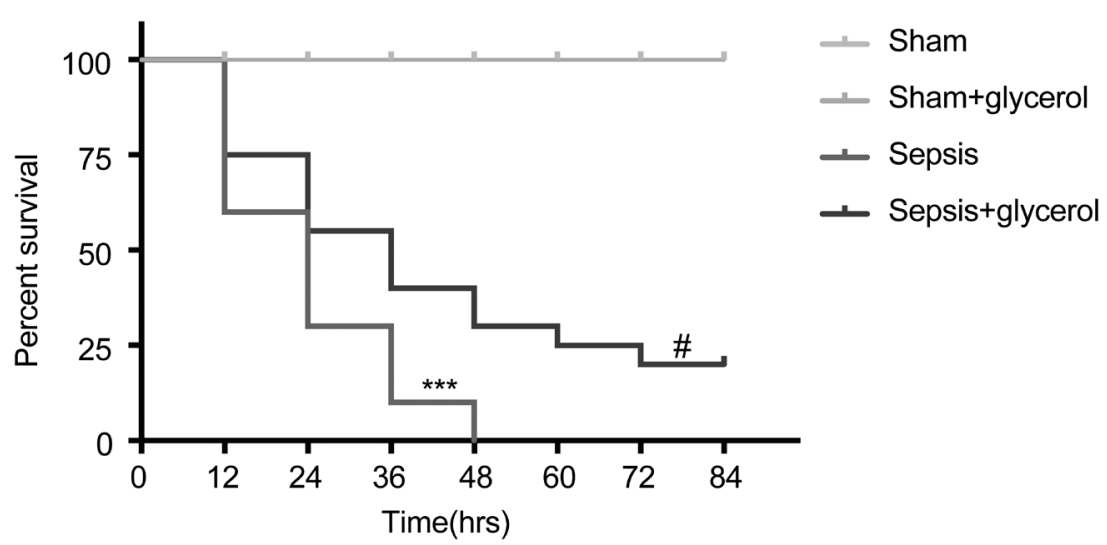

Fig. 1. Comparison of Survival among the Four Groups

Glycerol treatment improved the survival of CLP-induced septic mice. However, oral glycerol treatment did not influence the survival rate of the Sham group (two lines merged into one). Kaplan-Meier survival curves for four groups of mice $84 \mathrm{~h}$ after CLP $(n=20)$. Black, purple, red and blue indicate the Sham, Sham + glycerol, Sepsis, and Sepsis + glycerol groups, respectively; ${ }^{* *} p<0.001$, versus Sham group; ${ }^{*} p<0.05$, versus Sepsis group.
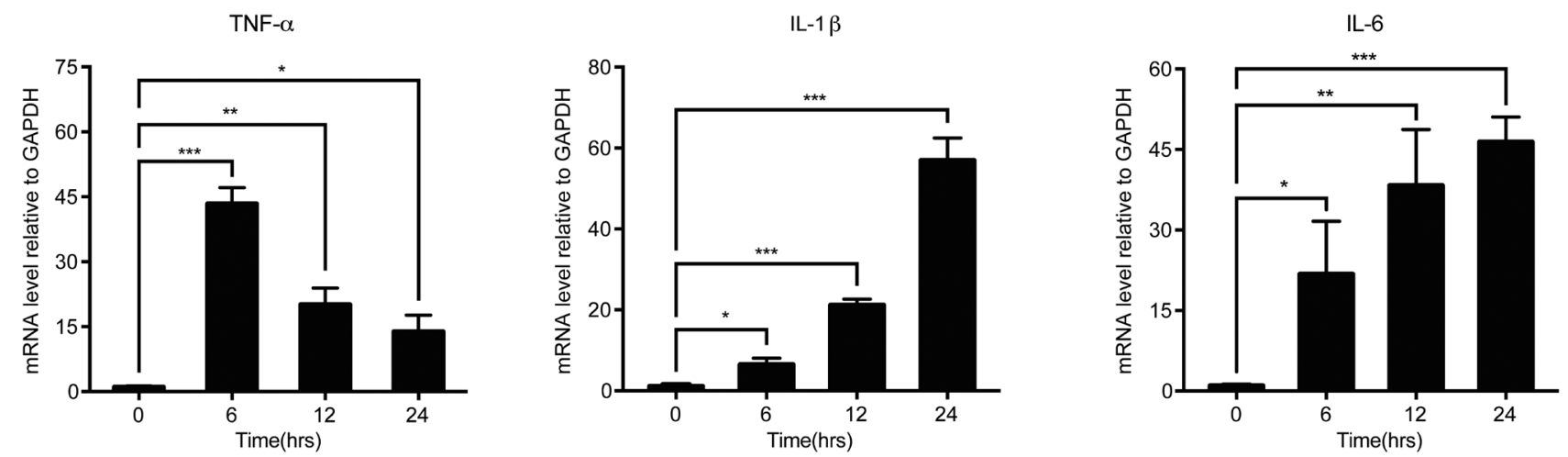

Fig. 2. The Expression Levels of TNF- $\alpha$, IL-1 $\beta$ and IL-6 Increased in Septic Animals

Intestinal mucosa was collected from the mice at $0,6,12$, and $24 \mathrm{~h}$ after CLP. qRT-PCR was used to detect the expression levels of TNF- $\alpha$, IL-1 $\beta$ and IL-6. The results are presented as the mean \pm S.D. $n=6$ mice per group. $* p<0.05 ; * * p<0.01 ; * * * p<0.001$.

effect relationship ( $p<0.05,0 \mathrm{~h} v s .6 \mathrm{~h}, 12 \mathrm{~h}$ or $24 \mathrm{~h}$ after CLP) and a peak at $24 \mathrm{~h}$ after CLP (Fig. 3B).

Both DAO and FABP2 were assessed as biomarkers of the integrity of the intestinal epithelia. ${ }^{14)}$ Plasma DAO (Fig. 3C) and FABP2 (Fig. 3D) concentrations increased $(p<0.05)$ as the disease progressed, and there were clear time-effect relationships.

Occludin plays a key role in maintaining the integrity of the intestinal barrier. Our results showed that the protein (Figs. 3E, F) expression levels of occludin in the intestinal mucosa decreased significantly with the increase in time postCLP compared with those at the time immediately after CLP $(p<0.001,0 \mathrm{~h})$. Taken together, the data presented in Fig. 3 indicate that intestinal injury clearly occurred in septic mice.

AQP3 Was Downregulated in the Intestinal Epithelium during Sepsis Regulating the expression of AQP3 may induce injury to the intestinal mucosa and/or translocation of the intestinal flora. ${ }^{15,16)}$ To determine whether AQP3 is involved in sepsis-induced intestinal injury, immunohistochemistry, qRTPCR (Fig. 4B), and Western blotting were performed to detect AQP3 expression. The immunohistochemistry (Fig. 4A), Western blot (Figs. 4B, C), and qRT-PCR (Fig. 4D) results showed that sepsis induced a time-dependent decrease in the expression of AQP3. Compared with the expression levels immediately after CLP, there were significant reductions in the expression levels of AQP3 at 6, 12, and 24h after CLP. These data indicated that sepsis induced intestinal mucosal damage by inhibiting the expression of AQP3.

Glycerol Treatment Ameliorated the Intestinal Injury A glycerol replacement experiment was conducted to observe the protective effect of AQP3 on intestinal mucosa damaged by sepsis. The pathological analysis showed integrated intestinal mucosa and compactly arrayed epithelium in the mice of the Sham and Sham + glycerol groups. In contrast, the intestinal mucosal were clearly damaged, with microciliary atrophy, microciliary disruption and loss of apical epithelial cells in the mice in the Sepsis and Sepsis + glycerol groups $24 \mathrm{~h}$ after the induction of sepsis. Glycerol treatment partly ameliorated the pathological injury of the ileum, with improved villi height compared with that of the Sepsis group (Fig. 5A). Chiu's scores in mice in the Sepsis + glycerol group were significantly lower than those of the mice in the Sepsis group, which was inconsistent with the histopathological changes (Fig. 5B). To further verify the protective effect of oral glycerol substitution on sepsis-induced intestinal mucosa damage, biochemical experiments were performed. A similar pattern of changes in intestinal barrier function was noted. The plasma DAO and FABP2 levels were significantly increased after $24 \mathrm{~h}$ in the Sepsis group compared with the Sham group $(p<0.001)$. Glycerol treatment resulted in lower DAO and FABP2 levels at $24 \mathrm{~h}$ than those in the Sepsis group $(p<0.05)$ (Figs. 5C, D). Occludin plays a critical role in maintaining the integrity 
A

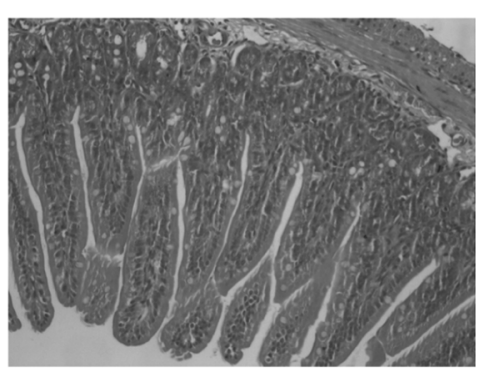

$0 \mathrm{hrs}$

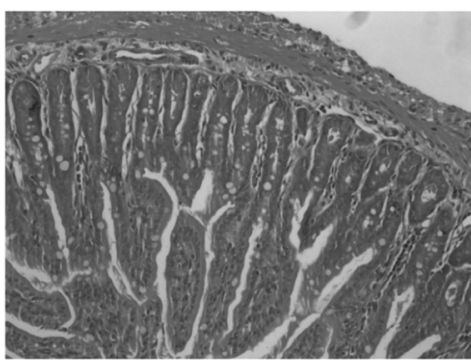

$12 \mathrm{hrs}$

D

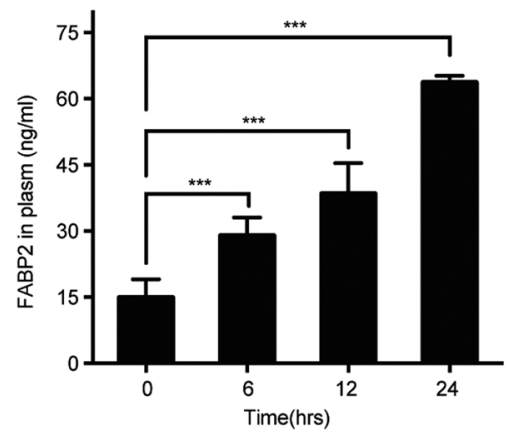

B

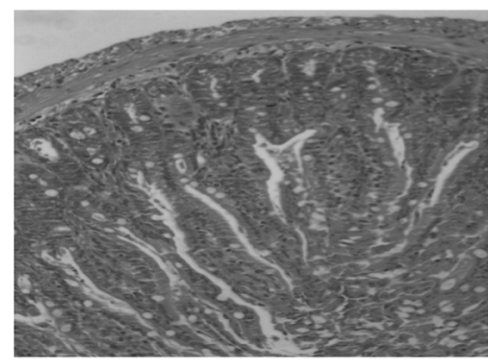

6 hrs

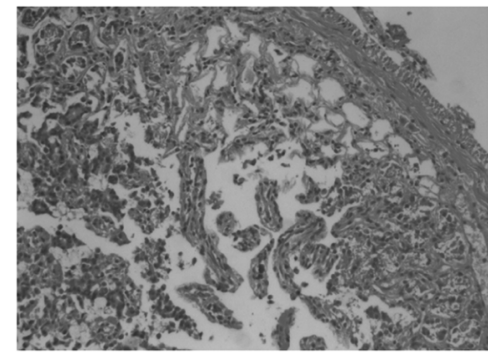

$24 \mathrm{hrs}$

E

$0 \mathrm{hrs} 6 \mathrm{hrs} 12 \mathrm{hrs} 24 \mathrm{hrs}$

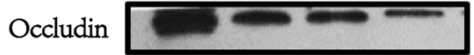

$\beta$-actin

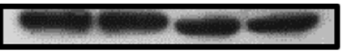

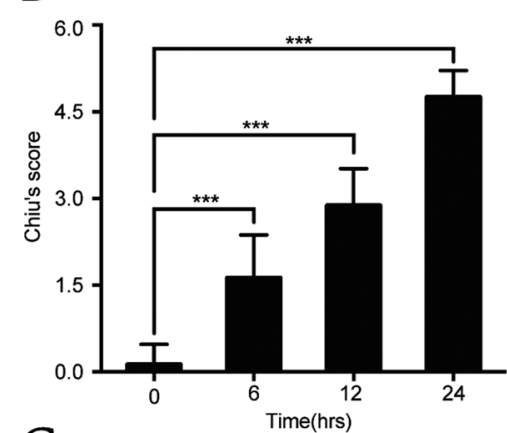

C

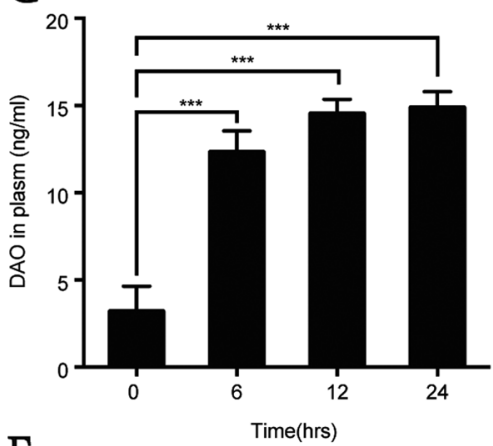

F

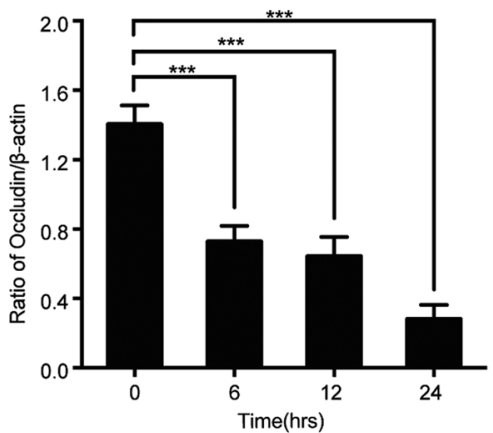

Fig. 3. Sepsis Induced Intestinal Barrier Injury

Intestinal tissue was collected from the mice at $0,6,12$, and $24 \mathrm{~h}$ after CLP. (A) Representative images of intestinal morphology (hematoxylin-eosin staining, magnification $\times 200$ ). (B) Intestinal mucosa injury was quantitative assessed by Chiu's score. (C, D) Plasma concentrations of DAO and IFAB2 (markers of intestinal permeability) were measured using ELISA. (E, F) Western blot was used to detect the expression of occludin. DAO and IFAB2 concentrations are expressed as the mean \pm S.D., $n=6$ per group. ${ }^{*} p<0.05, * * p<0.01, * * * p<0.001$, versus $0 \mathrm{~h}$ after CLP.

of the intestinal barrier. Our results showed that there was no significant difference between the Sham and Sham + glycerol groups in terms of the mRNA and protein expression levels of occludin $(p>0.05)$; however those levels were significantly lower in the Sepsis groups than in the Sham group (Sham vs. Sepsis, or Sham vs. Sepsis + glycerol, $p<0.01$ ). In contrast, glycerol treatment restored the inhibition of occludin expression to some extent (Sepsis vs. Sepsis + glycerol, $p<0.05$ ). (Figs. 5E-G)

\section{DISCUSSION}

Sepsis induced intestinal mucosal destruction, intestinal wall congestion and necrosis are the main causes of intestinal dysfunction. In this study, mice sepsis model was replicated by CLP, after which, intestinal fistula was formed, bacteria multiplied rapidly and endotoxin was produced in large quantities, Therefore, sepsis occurred rapidly and severely. ${ }^{17)}$ Intestinal bacteria are abundant, affecting the occurrence and development of critical illness. When the body is in a stress state, the redistribution of systemic blood flow and the decrease of gastrointestinal blood flow significantly lead to the damage of intestinal mucosa and villous cells, the loss of intestinal barrier function and the translocation of intestinal bacteria and toxins. In this study, after CLP, mucosal morphology was damaged, showing that intestinal mucosal villi were obviously damaged, with apical epithelial cells of villi missing, and microvilli thinner and shorter.

To further validate the sepsis model in the present study, the expression levels of TNF- $\alpha$, IL- 6 and IL- $1 \beta$, which are important inflammatory mediators of sepsis, were detected. Among them, the expression level of TNF- $\alpha$ was significantly increased in the early stage of the disease. ${ }^{18)}$ Our experiments showed that TNF- $\alpha$ peaked $6 \mathrm{~h}$ after CLP and then decreased. In contrast, IL- 6 and IL- $1 \beta$ progressively increased in a timedependent manner and then peaked $24 \mathrm{~h}$ after CLP. IL- 6 , as the main secondary inflammatory mediator, can inhibit the synthesis of TNF- $\alpha$ induced by endotoxin. Thus, its level might reflect the severity and prognosis of sepsis, ${ }^{19,20)}$ which is consistent with findings in the present study. 
A
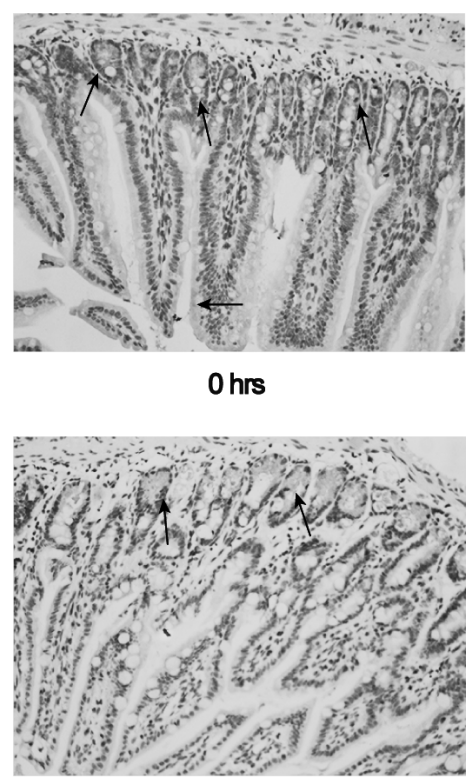

12 hrs

B

Ohrs 6hrs 12hrs 24hrs

AQP3

$\beta$-actin $\square$

$\mathrm{D}$

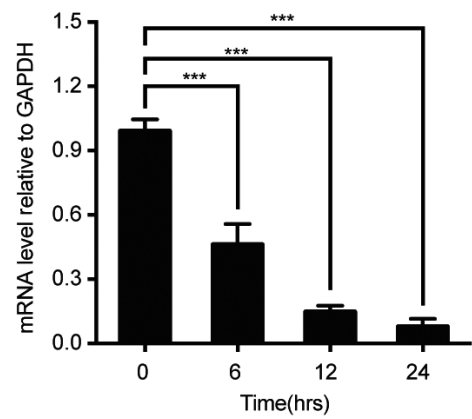

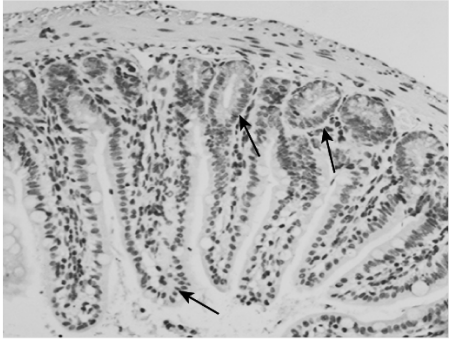

$6 \mathrm{hrs}$

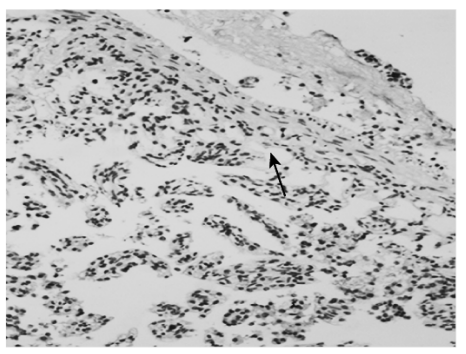

24 hrs

C

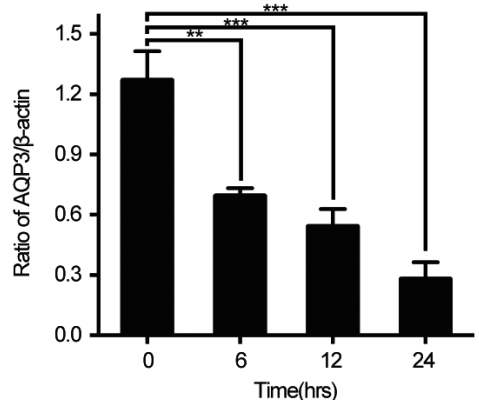

Fig. 4. Sepsis Induced AQP3 Downregulation in the Intestinal Epithelium

(A) Immunohistochemical staining for AQP3 in intestinal tissue sections of septic mice at different times (magnification $\times 200$ ). (B-D) qRT-PCR and Western blot were used to detect the expression of AQP3. Sepsis inhibited the expression of AQP3 in a time-dependent manner. The graph represents the relative band densities. The data are presented as the mean \pm S.D., $n=6$ per group. ${ }^{*} p<0.05, * * p<0.01, * * * p<0.001$, versus the $0 \mathrm{~h}$ group.

DAO is a highly active intracellular enzyme, which mostly exists in small intestinal mucosal villi. Its activity is closely related to the height of villi and the synthesis of nucleic acid and protein in mucosal cells. DAO is one of the sensitive indicators reflecting the structure and function of small intestinal mucosa. ${ }^{14,15,21)}$ The changes of DAO level in both tissues and plasma come from two ways: one is that necrotic intestinal mucosal cells fall into the intestinal cavity, which leads to the decrease of DAO activity in intestinal mucosa; the other is that DAO in the intestinal cavity enters the lymphatic vessels and capillaries in the intestinal interstitial space, which leads to the increase of DAO level in the blood. Therefore, the function of intestinal mucosal barrier can be reflected by measuring the level of DAO in blood and intestinal tissue. FABP2 is a unique low-molecular-weight protein in the cytoplasm of intestinal mucosa of mammals, which mainly locates at the tip of intestinal mucosa microvilli and has high tissue specificity. Even in the early stage of intestinal ischemia, only mucosa is involved and intestinal epithelial cell integrity is destroyed, FABP2 is released into the blood. Studies have shown that 
A
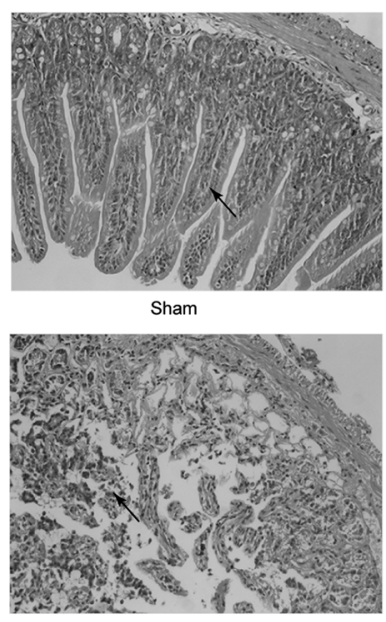

Sepsis

C

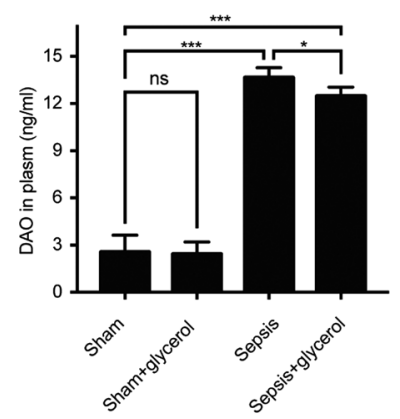

F

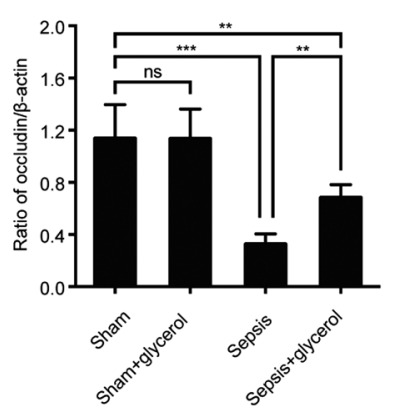

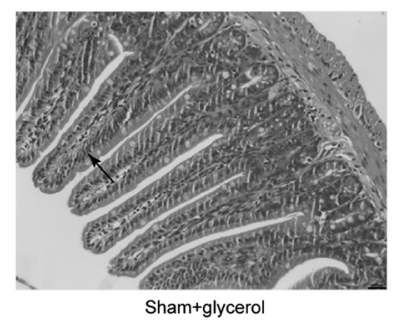

Sham+glycerol

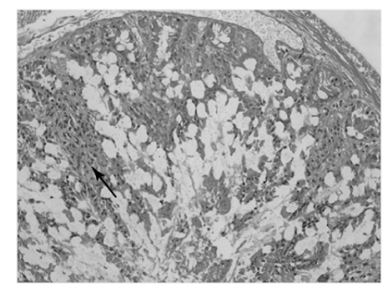

Sepsis+glycerol

$\mathrm{D}$

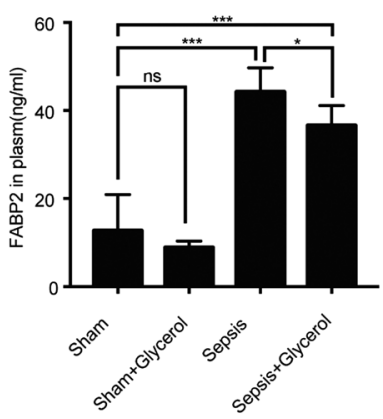

G

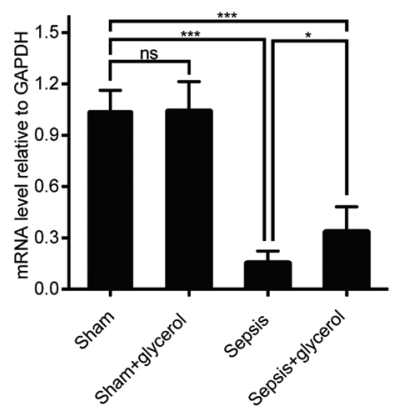

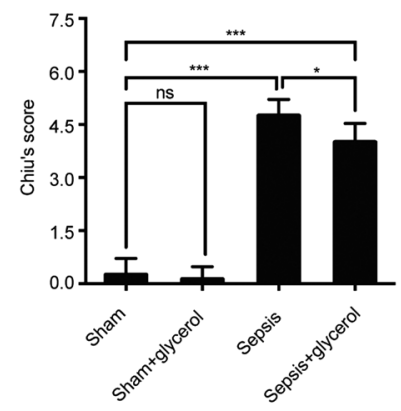

E
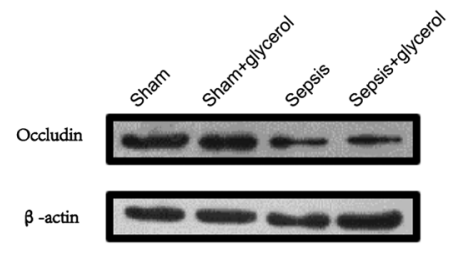

Fig. 5. Glycerol Treatment Alleviated Intestinal Injury Induced by CLP

The administration of oral glycerol partially alleviated the sepsis-induced injury of the intestinal mucosa. (A) Microscopic findings of the intestines stained with H\&E $24 \mathrm{~h}$ after CLP in the Sham, Sham + glycerol, Sepsis and Sepsis + glycerol groups (magnification $\times 200)$. (B) Intestinal mucosa injury was assessed by Chiu's score. (C, D) ELISAs were used to measure the plasma concentrations of DAO and FABP2. The levels of both DAO and FABP2 were lower in the Sepsis + Glycerol group than in the Sepsis group. (E-G) qRT-PCR and Western blot were used to detect the expression of occludin. The oral glycerol treatment restored the inhibition of occludin expression. ${ }^{\#} p<0.05$, versus CLP, $* * * p<0.001$, versus Sham; ns indicates no significance. The data are presented as the mean \pm S.D., $n=6$ per group. $* p<0.05, * * p<0.01$, $* * * p<0.001$, ns indicates no significance.

plasma FABP2 level can be used as an indicator of early intestinal mucosal damage and ischemia. ${ }^{22,23)}$ In this study, the levels of DAO and FABP2 in plasma increased with the severity of intestinal damage in sepsis mice, suggesting that intestinal mucosal damage and permeability increase.

The intestinal tract is the "motor" of MODS, ${ }^{24)}$ so the recovery of intestinal barrier function is one of the most important therapeutic strategies for sepsis. AQP3 is an aquaporin that transports water, glycerol and small solutes through the plasma membrane; its function is not limited to liquid transport but also involves cell proliferation, migration, ${ }^{25)}$ skin hydration, ${ }^{26)}$ wound healing, ${ }^{27)}$ and tumorigenesis. ${ }^{28)}$ There is a high level of AQP3 expression in the intestinal tract, ${ }^{9)}$ regu- lating the absorption and secretion of liquid and maintaining the steady state. Previous studies have shown that targeted regulation of AQP3 can induce intestinal injury and intestinal bacterial translocation. ${ }^{15,16)}$

The main characteristics of sepsis are the initial storm of inflammatory factors, damage of intestinal epithelial cells and increased barrier permeability, which eventually lead to multiple organ dysfunction. AQP3 decreased significantly in the lower part of the intestine ${ }^{29}$ of inflammatory bowel disease, indicating that intestinal inflammation and water/solute homeostasis are related to the abnormal regulation of AQP3, leading to transport dysfunction and participating in the main pathological mechanism of intestinal inflammation. Exces- 
sive inflammation leads to intestinal injury and deficiency of AQP3, which interferes with the migration of intestinal immune cells, ${ }^{30}$ ) leading to further expansion of inflammation, aggravating intestinal damage and forming a vicious circle. AQP3 is involved in inflammatory immunity of intestine and can regulate intestinal resistance. Wang et al. reported that heat shock (HS)-induced small intestinal barrier damage was caused by the disordered regulation of AQPs, ${ }^{31)}$ which might lead to bacterial translocation and systemic infection. Clearly, the disturbance of intestinal AQPs may be an important target for intestinal injury and bacterial translocation. Zhi et al. ${ }^{15)}$ showed that regulation of AQP3 by miR-874 led to intestinal barrier dysfunction and even MODS in patients with sepsis. On the other hand, the IncRNA H19, a competitive endogenous RNA, regulates the expression of AQP3 through competition with miR-874 and ameliorates intestinal barrier injury. ${ }^{16)}$ In mice with colitis, colonic bleeding, the loss of epithelial cells, cell death and other phenomena were more severe in AQP3 knockout mice with colonic injuries. Our study found that the expression of AQP3 was down-regulated after CLP in a time-dependent manner. At the same time, the intestinal mucosa was damaged and permeability increased. It is not clear that intestinal mucosal injury leads to a decrease in AQP3 expression, or vice versa, or that both may result from each other. The aim of this study was to explore whether AQP3 has protective effect on intestinal mucosal barrier in sepsis.

The repair of intestinal epithelial cells is a necessary process for the repair of intestinal mucosal barrier function after injury. The timely repair of intestinal mucosal mechanical barrier will play an important role in the protection of intestinal function after intestinal injury. ${ }^{32,33)}$ Studies showed that intestinal AQP3 was essential for the proliferation and repair of intestinal epithelial cells. ${ }^{12,27)}$ Thiagarajah et al. ${ }^{12)}$ showed that oral glycerol could partly replace the function of AQP3. Oral glycerol not only reduced the death of colon cells, but also saved cell proliferation damage. ${ }^{12)}$ Besides, in AQP3 knockout mice, glycerol replacement therapy significantly improved skin hydration, elasticity and barrier function. ${ }^{34)}$ However, the use of physicochemical glycerol-like substances such as sandalwood, erythritol or propylene glycol does not improve the hydration of the skin and does not alleviate skin dryness even with increased environmental humidity. ${ }^{34)}$ It can be seen that glycerol is essential for the important role of AQP3. Based on previous studies, in our study, oral glycerol was given before CLP to mimic the protective effect of AQP3 on sepsis-induced intestinal mucosal damage. The results showed that glycerol treatment partly ameliorated the pathological injury of small intestine, with orderly arrangement of villi and the increase of height; while plasma levels of DAO and FABP2 decreased, suggesting that glycerol might replace AQP3, reduce the permeability of intestinal epithelial cells and alleviate the damage of intestinal mucosa. It was also found that oral glycerol could reduce the mortality of mice. So far, the specific mechanism to the protective effect of glycerol on intestinal mucosa has not been clarified, which may be the direct protective agent of mucosa, or it may play a role by inhibiting the down-regulation of AQP3.

One way to repair the barrier and protect the body from gut microbes is to rely on hydrogen peroxide produced outside the epithelium monolayer cells. Thiagarajah and other researchers have shown that AQP3 transports hydrogen peroxide $\left(\mathrm{H}_{2} \mathrm{O}_{2}\right)$. In the process of intestinal injury stress and/or intestinal bacterial stimulation, AQP3 knockout mice showed destroyed lamellar foot; impaired colonic epithelial cell adhesion and repair ability, manifested by delayed healing of colon injury; and impaired mucosal innate immune responses against gut bacteria. ${ }^{35)}$ Our current results indicated that in sepsis-induced intestinal injury, the disordered mucosal structure and intestinal barrier damage were more serious when sepsis was aggravated. Meanwhile, the expression levels of AQP3 and occludin were significantly downregulated. Surprisingly, the glycerol treatment partially alleviated intestinal damage and reduced mortality in septic mice despite severe intestinal injury and a low survival rate. This may be due to the following reasons. First, sepsis is characterized by a high degree of intestinal injury, high mortality and difficult treatment. Second, the glycerol treatment cannot completely replace the role of AQP3, which is reflected in the fact that glycerol treatment has no effect on the transport of hydrogen peroxide. As mentioned above, the transport of hydrogen peroxide is an important mechanism by which AQP3 regulates intestinal oxidative stress and repairs intestinal injury. The specific mechanism of oral glycerol substituting AQP3 needs further study.

It was found that after CLP, sepsis mice showed different degrees of diarrhea, such as mucous stool or bloody stool, in addition to decreased activity, depression and abdominal incision exudation. Ikarashi et al. reported that the expression of AQP3 in the colon was associated with diarrhea and constipation, ${ }^{36)}$ while Zhang et al. disclosed that the expression of AQP3 in the small intestine was down-regulated during enterotoxigenic Escherichia coli-induced diarrhea in mice. ${ }^{37}$ Kon et al. recently found that anti-inflammatory drugs that suppress the reduction of AQP3 expression could prevent CPT11-induced delayed diarrhea. In this present study the mice showed mucus stool, which may be due to the reduced expression of AQP3 in the large intestine. ${ }^{38)}$

In this study, only AQP3 was concerned, and no other AQPs were involved. Gastrointestinal mucosa is second only to kidney in water conversion. Previous literature reported that aquaporin 1, 4, 8 knockout mice had no significant effect on intestinal fluid transport function ${ }^{39-41)}$; AQP7 deficiency led to fat cell hypertrophy and glycerol accumulation ${ }^{42)}$; AQP3 deficiency led to intestinal cell proliferation damage and even diarrhea in enteritis mice. The further studies will involve the effects of sepsis or on $t$ oral glycerin on the expression of AQP family in small intestine or large intestine tissues. There are also some limitations in this study. Firstly, glycerol substitution test was used to study the protective effect of AQP3 on intestinal mucosa of sepsis. The results showed that the intestinal mucosal injury of sepsis could only be partially alleviated. Further studies using AQP3 gene knockout technology could clarify the protective effect of AQP3 on intestinal mucosa more clearly. Secondly, intestinal mucosal barrier function includes mechanical barrier, immune barrier and chemical barrier stress biological barrier. In addition, the effects of AQP3 on oxidative stress and inflammatory factor migration in intestinal mucosa of sepsis need to be further studied to provide more evidence for the protective effect of AQP3 on intestinal mucosa. The expressions of AQP3 and occudin were detected in whole intestinal tissue rather than isolated intestinal epithelial cells. Inflammation or apoptosis 
in sepsis may cause intestinal mucosal epithelial cell death, leading to the decrease of their expression. This is another shortcoming of this study.

In summary, the intestinal mucosa of sepsis mice was damaged, plasma DAO and FABP2 levels were increased, and the expression of AQP3 in intestinal mucosa was down-regulated. Glycerol substitution test can partly replace AQP3 and reduce the pathological damage of intestinal tissue. However, there are some limitations in glycerol substitution test, which cannot fully explain the role of AQP3 in septic intestinal injury, and its mechanism needs further study.

\section{CONCLUSION}

This study confirmed that the expression of AQP3 was downregulated in sepsis intestinal injury, and the downregulation of the expression was significantly greater when the disease was more severe. Glycerol was a partial substitute for AQP3 and was able to reduce the pathological damage to intestinal tissues and even improve the survival rate. However, glycerol substitution has some limitations, and the glycerol substitution experiment could not fully explain the role of AQP3 in septic intestinal injury; the specific mechanisms need to be further studied. Surprisingly, this study suggests that AQP3 may be involved in intestinal damage caused by sepsis, but it's protective mechanism of AQP3 on intestinal tract is still worthy of further study.

Acknowledgments This study was funded by the Zhejiang Public Welfare Technology Application Research Project (2017C37160), Zhejiang province health department project (2018KY173), Shaoxing Public Welfare Technology Application Research Project (2017B70038), National Natural Science Foundation of China (81771194).

Conflict of Interest The authors declare no conflict of interest.

\section{REFERENCES}

1) Singer M, Deutschman CS, Seymour CW, Shankar-Hari M, Annane D, Bauer M, Bellomo R, Bernard GR, Chiche JD, Coopersmith CM, Hotchkiss RS, Levy MM, Marshall JC, Martin GS, Opal SM, Rubenfeld GD, van der Poll T, Vincent JL, Angus DC. The third international consensus definitions for sepsis and septic shock (Sepsis-3). JAMA, 315, 801-810 (2016).

2) Hassan U, Ghonge T, Reddy B Jr, Patel M, Rappleye M, Taneja I, Tanna A, Healey R, Manusry N, Price Z, Jensen T, Berger J, Hasnain A, Flaugher E, Liu S, Davis B, Kumar J, White K, Bashir R. A point-of-care microfluidic biochip for quantification of CD64 expression from whole blood for sepsis stratification. Nat. Commun., 8, 15949 (2017).

3) Vakkalanka JP, Harland KK, Swanson MB, Mohr NM. Clinical and epidemiological variability in severe sepsis: an ecological study. $J$. Epidemiol. Community Health, 72, 741-745 (2018).

4) Vincent JL, Lefrant JY, Kotfis K, Nanchal R, Martin-Loeches I, Wittebole X, Sakka SG, Pickkers P, Moreno R, Sakr Y. Comparison of European ICU patients in 2012 (ICON) versus 2002 (SOAP). Intensive Care Med., 44, 337-344 (2018).

5) Barichello $T$, Sayana $P$, Giridharan VV, Arumanayagam AS, Narendran B, Della Giustina A, Petronilho F, Quevedo J, Dal-Pizzol F. Long-term cognitive outcomes after sepsis: a translational systematic review. Mol. Neurobiol., 56, 186-251 (2019).
6) Meng M, Klingensmith NJ, Coopersmith CM. New insights into the gut as the driver of critical illness and organ failure. Curr. Opin. Crit. Care, 23, 143-148 (2017).

7) Mittal R, Coopersmith CM. Redefining the gut as the motor of critical illness. Trends Mol. Med., 20, 214-223 (2014).

8) Kagnoff MF. The intestinal epithelium is an integral component of a communications network. J. Clin. Invest., 124, 2841-2843 (2014).

9) Zhu C, Chen Z, Jiang Z. Expression, distribution and role of aquaporin water channels in human and animal stomach and intestines. Int. J. Mol. Sci., 17, 1399 (2016).

10) Rump K, Adamzik M. Function of aquaporins in sepsis: a systematic review. Cell Biosci, 8, 10 (2018).

11) Buras JA, Holzmann B, Sitkovsky M. Animal models of sepsis: setting the stage. Nat. Rev. Drug Discov., 4, 854-865 (2005).

12) Thiagarajah JR, Zhao D, Verkman AS. Impaired enterocyte proliferation in aquaporin-3 deficiency in mouse models of colitis. Gut, 56, 1529-1535 (2007).

13) Chiu CJ, McArdle AH, Brown R, Scott HJ, Gurd FN. Intestinal mucosal lesion in low-flow states. I. A morphological, hemodynamic, and metabolic reappraisal. Arch. Surg., 101, 478-483 (1970).

14) Chi X, Yao W, Xia H, Jin Y, Li X, Cai J, Hei Z. Elevation of HO-1 expression mitigates intestinal ischemia-reperfusion injury and restores tight junction function in a rat liver transplantation model. Oxid. Med. Cell. Longev., 2015, 986075 (2015).

15) Zhi X, Tao J, Li Z, Jiang B, Feng J, Yang L, Xu H, Xu Z. MiR-874 promotes intestinal barrier dysfunction through targeting AQP3 following intestinal ischemic injury. FEBS Lett., 588, 757-763 (2014).

16) Su Z, Zhi X, Zhang Q, Yang L, Xu H, Xu Z. LncRNA H19 functions as a competing endogenous RNA to regulate AQP3 expression by sponging miR-874 in the intestinal barrier. FEBS Lett., 590, 1354-1364 (2016).

17) Buras JA, Holzmann B, Sitkovsky M. Animal models of sepsis: setting the stage. Nat. Rev. Drug Discov., 4, 854-865 (2005).

18) Mera S, Tatulescu D, Cismaru C, Bondor C, Slavcovici A, Zanc V, Carstina D, Oltean M. Multiplex cytokine profiling in patients with sepsis. APMIS, 119, 155-163 (2011).

19) Chawla LS, Seneff MG, Nelson DR, Williams M, Levy H, Kimmel PL, Macias WL. Elevated plasma concentrations of IL-6 and elevated APACHE II score predict acute kidney injury in patients with severe sepsis. Clin. J. Am. Soc. Nephrol., 2, 22-30 (2007).

20) Cavaillon JM, Annane D. Compartmentalization of the inflammatory response in sepsis and SIRS. J. Endotoxin Res., 12, 151-170 (2006).

21) Li Y, Ren J, Wu X, Li J. Intra-abdominal infection combined with intra-abdominal hypertension aggravates the intestinal mucosal barrier dysfunction. Biosci. Rep., 38, BSR20170931 (2018).

22) Lau E, Marques C, Pestana D, Santoalha M, Carvalho D, Freitas $\mathrm{P}$, Calhau $\mathrm{C}$. The role of I-FABP as a biomarker of intestinal barrier dysfunction driven by gut microbiota changes in obesity. Nutr. Metab. (Lond.), 13, 31 (2016).

23) Treskes N, Persoon AM, van Zanten ARH. Diagnostic accuracy of novel serological biomarkers to detect acute mesenteric ischemia: a systematic review and meta-analysis. Intern. Emerg. Med., 12, 821-836 (2017).

24) Dickson RP. The microbiome and critical illness. The Lancet Respiratory Medicine, 4, 59-72 (2016).

25) Galán-Cobo A, Ramirez-Lorca R, Echevarria M. Role of aquaporins in cell proliferation: What else beyond water permeability? Channels (Austin), 10, 185-201 (2016)

26) Matsunaga $N$, Itcho $K$, Hamamura $K$, Ikeda E, Ikeyama H, Furuichi Y, Watanabe M, Koyanagi S, Ohdo S. 24-hour rhythm of aquaporin-3 function in the epidermis is regulated by molecular clocks. $J$. Invest. Dermatol., 134, 1636-1644 (2014).

27) Sebastian R, Chau E, Fillmore P, Matthews J, Price LA, Sidhaye V, Milner SM. Epidermal aquaporin-3 is increased in the cutaneous burn wound. Burns, 41, 843-847 (2015). 
28) Nagaraju GP, Basha R, Rajitha B, Alese OB, Alam A, Pattnaik S, El-Rayes B. Aquaporins: Their role in gastrointestinal malignancies. Cancer Lett., 373, 12-18 (2016).

29) Ricanek P, Lunde LK, Frye SA, Stoen M, Nygard S, Morth JP, Rydning A, Vatn MH, Amiry-Moghaddam M, Tonjum T. Reduced expression of aquaporins in human intestinal mucosa in early stage inflammatory bowel disease. Clin. Exp. Gastroenterol., 8, 49-67 (2015).

30) Hara-Chikuma M, Chikuma S, Sugiyama Y, Kabashima K, Verkman AS, Inoue S, Miyachi Y. Chemokine-dependent T cell migration requires aquaporin-3-mediated hydrogen peroxide uptake. $J$. Exp. Med., 209, 1743-1752 (2012).

31) Wang YH, Liu TT, Kung WM, Chen CC, Wen YT, Lin IC, Huang CC, Wei L. Expression of aquaporins in intestine after heat stroke. Int. J. Clin. Exp. Pathol., 8, 8742-8753 (2015).

32) Alam A, Leoni G, Wentworth CC, Kwal JM, Wu H, Ardita CS, Swanson PA, Lambeth JD, Jones RM, Nusrat A, Neish AS. Redox signaling regulates commensal-mediated mucosal homeostasis and restitution and requires formyl peptide receptor 1. Mucosal Immunol., 7, 645-655 (2014).

33) Leoni G, Neumann PA, Kamaly N, Quiros M, Nishio H, Jones HR, Sumagin R, Hilgarth RS, Alam A, Fredman G, Argyris I, Rijcken E, Kusters D, Reutelingsperger C, Perretti M, Parkos CA, Farokhzad OC, Neish AS, Nusrat A. Annexin A1-containing extracellular vesicles and polymeric nanoparticles promote epithelial wound repair. J. Clin. Invest., 125, 1215-1227 (2015).

34) Hara M, Verkman AS. Glycerol replacement corrects defective skin hydration, elasticity, and barrier function in aquaporin-3-deficient mice. Proc. Natl. Acad. Sci. U.S.A., 100, 7360-7365 (2003).

35) Thiagarajah JR, Chang J, Goettel JA, Verkman AS, Lencer WI. Aquaporin-3 mediates hydrogen peroxide-dependent responses to environmental stress in colonic epithelia. Proc. Natl. Acad. Sci. U.S.A., 114, 568-573 (2017).

36) Ikarashi N, Kon R, Sugiyama K. Aquaporins in the colon as a new therapeutic target in diarrhea and constipation. Int. J. Mol. Sci., 17, 1172 (2016).

37) Zhang D, Zhang K, Su W, Zhao Y, Ma X, Qian G, Qu G, Pei Z, Liu $\mathrm{S}, \mathrm{Ma} \mathrm{H}$. Aquaporin-3 is down-regulated in jejunum villi epithelial cells during enterotoxigenic Escherichia coli-induced diarrhea in mice. Microb. Pathog., 107, 430-435 (2017).

38) Kon R, Tsubota Y, Minami M, Kato S, Matsunaga Y, Kimura H, Murakami Y, Fujikawa T, Sakurai R, Tomimoto R, Machida Y, Ikarashi N, Sugiyama K. CPT-11-induced delayed diarrhea develops via reduced aquaporin-3 expression in the colon. Int. J. Mol. Sci., 19, 170-185 (2018)

39) Mennone A, Verkman AS, Boyer JL. Unimpaired osmotic water permeability and fluid secretion in bile duct epithelia of AQP1 null mice. Am. J. Physiol. Gastrointest. Liver Physiol., 283, G739-G746 (2002).

40) Wang KS, Ma T, Filiz F, Verkman AS, Bastidas JA. Colon water transport in transgenic mice lacking aquaporin-4 water channels. Am. J. Physiol. Gastrointest. Liver Physiol., 279, G463-G470 (2000).

41) $\overline{\text { Yang B }}$, Song Y, Zhao D, Verkman AS. Phenotype analysis of aquaporin-8 null mice. Am. J. Physiol. Cell Physiol., 288, C1161-C1170 (2005).

42) Hara-Chikuma M, Sohara E, Rai T, Ikawa M, Okabe M, Sasaki S, Uchida S, Verkman AS. Progressive adipocyte hypertrophy in aquaporin-7-deficient mice: adipocyte glycerol permeability as a novel regulator of fat accumulation. J. Biol. Chem., 280, 15493-15496 (2005). 